\title{
Wireless Power Transmission: State of the Art and Perspectives
}

\author{
J. P. Fanton
}

\begin{abstract}
This paper deals with Wireless Power Transmission (WPT), and its main futuristic application, Space-based Solar Power (SSP). In the first part, the decisive role played by physicist Nikola TESLA in the development of electricity industry, his major concrete contributions, and his prophetic intuitions concerning wireless power are presented. The basics of the physics of the domain are also recalled. In the second part, the main experiments led until now in the domain of WPT are presented, as well as the different technical approaches available in the field. A few current applications of WPT at low power are briefly described. In the third part, a survey of the actual state of SSP projects is presented. Copyright (C) 2019 The Authors.

Published by Praise Worthy Prize S.r.l. This article is open access published under the CC BY-NC-ND license (http://creativecommons.org/licenses/by-nc-nd/3.0/).
\end{abstract}

Keywords: Harvesting, Rectenna, Space-Based Solar Power, Wireless Power Transmission

\section{Nomenclature}

Symbols

$\nabla \wedge$

$\nabla$.

$B$

$D$

E

$E$

$f, v$

$H$

I

j

$m$

$n_{1}, n_{2}$

$P$

$r$

$S_{p}$

$U$

Z

$\Phi$

$\chi$

$\lambda$

$\rho_{a}$

$\sigma$

$\omega$

Acronyms

$\mathrm{AC}$

DC

EN

ESA

FET

FSS

GEO
Curl operator

Divergence operator

Magnetic induction field in $\mathrm{V} \mathrm{s} \mathrm{m}^{-2}$

Electric induction field in $\mathrm{A} \mathrm{s} \mathrm{m}^{-2}$

Electric field in $\mathrm{V} \mathrm{m}^{-1}$

Electro Magnetic Force in $\mathrm{V}$

Frequency in $\mathrm{Hz}$

Magnetic field in $\mathrm{H} \mathrm{m}^{-1}$

Electric current in A

Current density in $\mathrm{A} \mathrm{m}^{-2}$

Transformer ratio (dimensionless)

Number of turns (dimensionless)

Power in W

Distance in $\mathrm{m}$

Poynting power density vector in $\mathrm{W} \mathrm{m}^{-2}$

Voltage in $\mathrm{V}$

Electrical impedance in $\Omega$

Magnetic induction flux in $\mathrm{Wb}$

Wave celerity in $\mathrm{m} \mathrm{s}^{-1}$

Electric permittivity in $\mathrm{F} \mathrm{m}^{-1}$

Angular phase in rad

Wavelength in $\mathrm{m}$

Magnetic permeability in $\mathrm{H} \mathrm{m}^{-1}$

Density of electric loads in A s m${ }^{-2}$

Electric conductivity in $\Omega^{-1} \mathrm{~m}^{-1}$

Pulsation in $\mathrm{rad} \mathrm{s}^{-1}$
Alternating Current
Direct Current
European Standard
European Space Agency
Field Effect Transistor
Frequency Selective Surface
Geostationary Earth Orbit

$\begin{array}{ll}\text { GTO } & \text { Gate Turn Off } \\ \text { IEC } & \begin{array}{l}\text { International Electrotechnical } \\ \text { Commission }\end{array} \\ \text { IEEE } & \begin{array}{l}\text { Institute of Electrical and Electronics } \\ \text { Engineers } \\ \text { Insulated Gate Bipolar Transistors }\end{array} \\ \text { ISM } & \begin{array}{l}\text { Industrial, Scientific, and Medical } \\ \text { applications frequency band }\end{array} \\ \text { JAXA } & \text { Japan Aerospace eXploration Agency } \\ \text { LEO } & \text { Low Earth Orbit } \\ \text { MEO } & \text { Medium Earth Orbit } \\ \text { MOS } & \text { Metal Oxide Semi Conductor } \\ \text { NASA } & \text { National Aeronautics and Space } \\ \text { NASDA } & \text { Administration } \\ \text { National Space Development Agency } \\ \text { PV } & \text { of Japan } \\ \text { RF } & \text { Photo Voltaic } \\ \text { RFID } & \text { Radio Frequency } \\ \text { RTE } & \text { Radio Frequency IDentifier } \\ \text { SERT } & \text { Réseau de Transport d'Électricité } \\ \text { SSP } & \text { Space Solar Power Exploratory } \\ \text { USA } & \text { Research and Technology } \\ \text { USAF } & \text { Space-based Solar Power } \\ \text { WPT } & \text { United States of America } \\ & \text { United States Air Force } \\ \text { Wireless Power Transmission }\end{array}$

\section{Introduction}

The transfer of electrical power without wires has for long been a dream for electrical engineers, at least from the end of the nineteenth century. The first experiments led at that time on this process have been unsuccessful; the process has thus been considered, until recently, as nearly hopeless. However, today, due to the technical and scientific progress in general, and more precisely due to the progress of electronic components, of modelling and 
experimentation capabilities, the topic finds a new interest, and applications begin to appear, among which some are already popular, such as mobile phone chargers and contactless credit cards. The goal of this paper is, after a few reminders about the birth of the Wireless Power Transmission (WPT) technique, to recall some physical principles, which determine the application domain accessible for WPT, in order to describe some significant existing applications and the undergoing research, and finally evoke the perspectives thus open for this technique.

\section{The Birth of WPT: the Tesla Projects and Expectations}

\section{II.1. Tesla: His Main Discoveries}

The technical field of electrical power transmission in general owes a considerable debt to inventor Nikola Tesla (1846-1943). The two principles universally enforced everywhere throughout the world today for energy wired transmission are in fact directly issued of his work:

a) the use of alternating current (AC) instead of direct current (DC) allows changing the level of the voltage, i.e. rising and decreasing it; this allows fighting the resistive Joule effect for energy transmission, on long distances and hence enables this transmission with acceptable thermal losses.

b) the conditioning of energy as multiphase systems (generally three-phase systems) allows the creation of rotating magnetic fields, and hence the operation of electrical rotating machinery (synchronous machines and induction machines). In other terms, it allows the electromechanical conversion of energy.

\section{II.1.1. Why Are These Inventions so Important?}

\section{Alternating current.}

It enables the transmission of electrical energy over long distances. This can happen thanks to the use of the transformer (see below), which allows easily raising or lowering the voltage level of a given source. Energy transmission under high voltage strongly decreases the power losses due to Joule resistive effect. In addition, AC enables easy evolution of the network, and efficient work of circuit breakers. It should be noted however that due to the progress of power electronics, DC can today be used for long distance high power transmission, using large-size converters. Although the corresponding "market share" of DC is increasing, and no longer limited to point-to-point links, DC will likely never replace AC in totality.

\section{Multiphase systems.}

They add new advantages to alternating current networks:

- Strong reduction of transmission losses by the reduction of the number of wires needed

- Instant power $\mathrm{P}(\mathrm{t})$ of generators remains constant:

$$
P(t)=q V I \cos j=C^{t}
$$

This property is essential for mechanical fatigue considerations applied to large turbo-generators.

- Possibility to create rotating magnetic fields

\section{The rotating magnetic fields.}

They open the way to power electrical machinery: synchronous machines and induction machines. Almost all high power mechanical drivers fed by electricity use either synchronous or induction machines. Most of the high power electrical generators also use these types of machines [1].

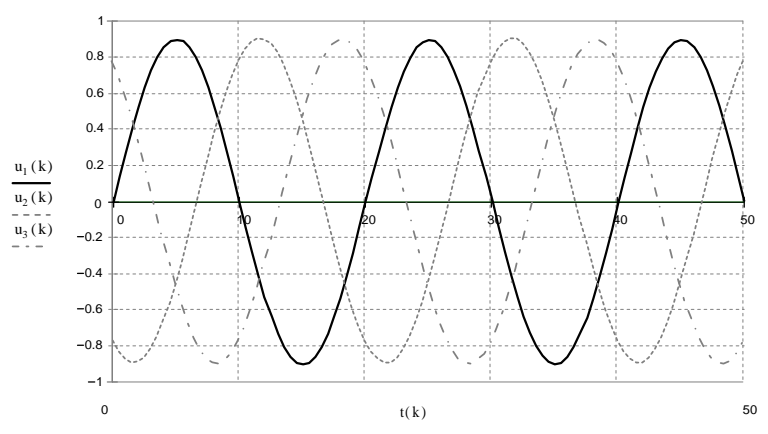

Fig. 1. A set of three-phased voltages

\section{II.2. Tesla's Other Theories and Experiments}

The work of Tesla goes far beyond his successful discoveries concerning alternating current and electrical energy wired networks. Arrived in the United States at the age of 28, he immediately started his work, in cooperation with Thomas Edison. After some time, a conflict arose between them, about the most efficient approach to electrification, conflict known as the "war of currents". Edison promoted Direct Current (DC), whereas Tesla, supported by George Westinghouse, advocated Alternating Current (AC). Edison made use of some unfair arguments, such as invoking the efficiency of the electric chair. Nevertheless, the solution of Tesla finally imposed itself, during a remarked demonstration in Chicago (1893). Tesla undertook an extended set of researches, in which he addressed the domains of broadcasting, X-rays, radar, and remote control of objects. His interest was equally shared between information transmission and energy transmission. His contribution to radio-transmission was acknowledged as prior to the one of G. Marconi, generally considered as the inventor of broadcasting, by the U.S. authorities. His main dream however has been wireless energy transmission, to which he devoted many years of his life.

Consequent efforts resulted, to implement demonstrative devices. A large wooden tower, intended for emission, was erected in Wardenclyffe, Long Island (Fig. 3). He postulated of the presence of energy everywhere, in the air and in the earth. The experiments he made were sometimes spectacular, due to the presence of very high voltage levels (hence also very large sparkles and artificial lightnings). 


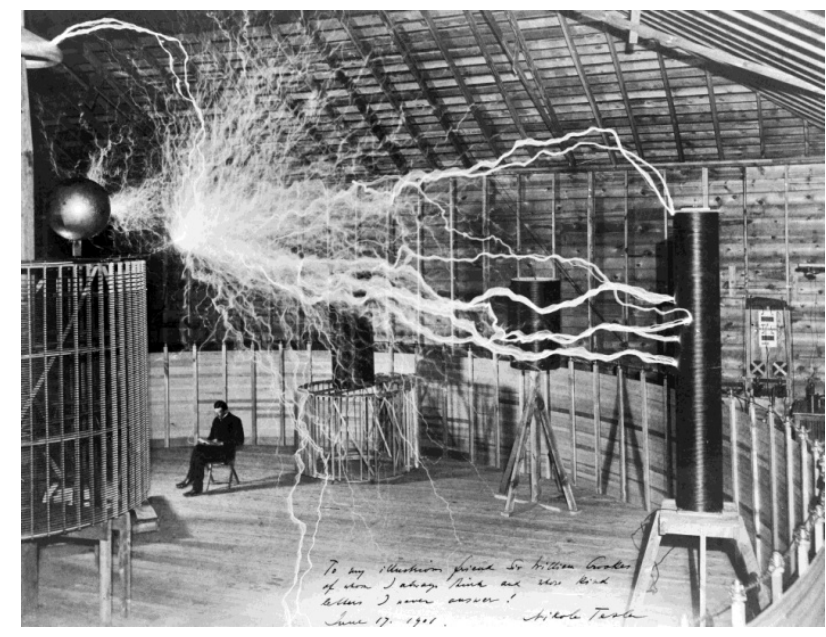

Fig. 2. Well-known, but doubtful, representation of Tesla in his laboratory of Colorado Springs

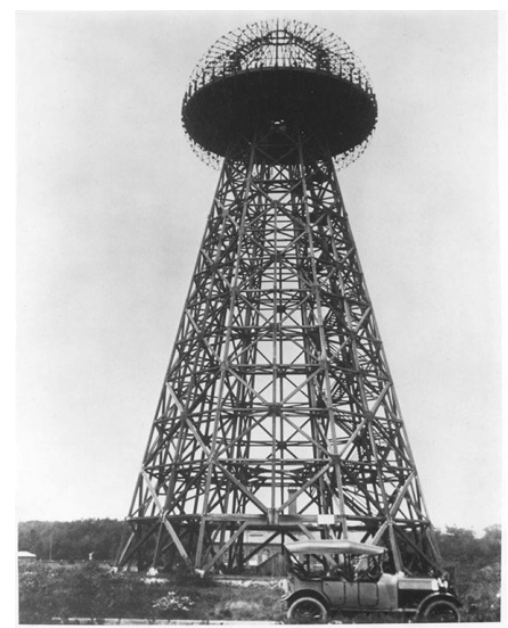

Fig. 3. The 57-m Wardenclyffe tower in Long Island was intended to allow large-scale experimentation on WPT

He obtained them by means of the famous "Tesla coil", still used today for toys and gadgets, which is simply a very inductive coil. The dielectric strength of dry air at normal pressure being around $3 \mathrm{MV}$ per meter, he likely obtained some hundreds of MV.

He otherwise succeeded in experiments using the earth conductive properties.Unfortunately during all these experiments no significant energy transfer could be established. Some authors have been unfair to him, suggesting he did not always understand what he did, and was sometimes acting at random. He would, on the contrary, have had a very fine sense of technical analysis.

At the time of Tesla's death, no significant results concerning WPT had yet been obtained.

\section{II.3. Tesla: His Personality}

Tesla ought to be considered today as one of the main contributors to the development of the modern industrial society, thank to his discoveries, as explained above. He was someone extremely creative. He held about 300 patents. A great part of his ideas and hypotheses could not be confirmed, such as those concerning free energy, or the existence of huge telluric currents and electric resonances in the earth. However, it is a commonplace in the world of scientific research that not all of the attempts of a researcher are followed by success. Tesla is an attaching figure. He experienced many difficulties during his career, lack of experimental means, accidental destruction of his laboratory; suspected as being a immigrant during World War I, he also suffered of diverse health problems such as lack of sleep and hallucinations. He always went forward anyway. He had some extraordinary intuitions, such as his belief in a world where everyone will immediately communicate.

He wrote : "Anyone, on land or at sea, with a simple and cheap device in his pocket, will receive news from around the world or messages intended only for the user." This prefigured the mobile phone and the web. He was a humanist. He placed the interest of science much beyond that of business. Despite all his activities, he finally died poor. With his work, Tesla has brought plenty to mankind. He does not need a legend ; truth is enough. However, he still keeps today a small part of mystery. He usually drove an electric car, which apparently contained no battery (It was not a Tesla car...), and all his technical papers were kept secret by U.S. authorities long after his death.

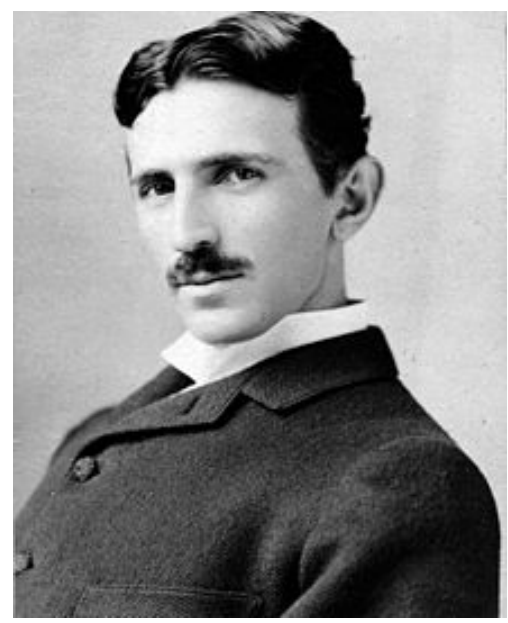

Fig. 4. A late portrait of Nikola Tesla

\section{Recall of Physics}

\section{III.1. Interactions due to Distant Forces}

Physics today identifies three types of distant interactions. By order of decreasing importance, interactions between particles are:

1) The strong interaction (intra-nuclear). It acts as the force of cohesion of the atomic nuclei. The associated particle is the gluon. This interaction takes place in the framework of nuclear energy, by either nuclei fission or fusion. At the time, only fission has applications for energy production.

2) The electromagnetic interaction. This interaction acts in an identical way at all scales (particle scale, human 
scale, cosmic scale). It relates to a very great number of physical fields: optics, acoustics, elasticity, electrochemistry, etc, that are the macroscopic demonstration of the electromagnetic interaction on a molecular scale. The particle associated with electromagnetism is the photon.

3) The weak interaction. This interaction is known as Fermi interaction, and today it is unified with the previous one. It is responsible for disintegration $\beta$, and it is represented in the particles domain by intermediate bosons.

4) The gravitational interaction. It acts as a mutual attraction between the masses. The corresponding particle could be only recently observed. Electrical power engineering, and hence WPT, are obviously interested in the second form of interaction.

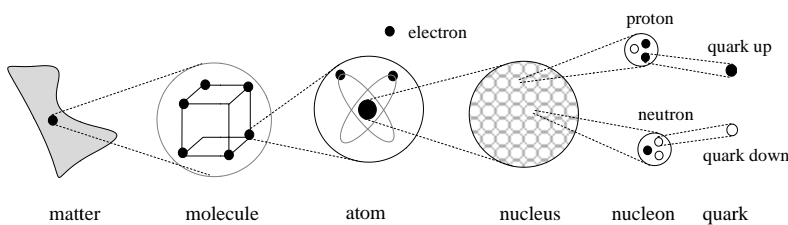

Fig. 5. The structure of matter

\section{III.2. Electricity and Magnetism}

Electricity and magnetism are in fact two observable forms of the sole electromagnetic interaction. Media and materials, either natural or artificial, can be classified as insulators or conductors, according to their behaviour with respect to the elementary electric charges present in the matter. These charges are constituted by the protons and the electrons; the mobile outer-shell electrons are known as conduction electrons. All electromagnetic phenomena are due to these charges. The electric phenomena result from the electro-kinetic displacement or from the electrostatic accumulation of these charges.

The electrical currents are generally the manifestation of the displacement of the conduction electrons in a conducting medium under the action of an electric field.

The magnetic phenomena can result, either from the action of an electrical current, (electro-magnetism) or from material properties (natural magnetism). In all the cases, they are due to the inner-shell mobility of the electrons inside the atoms (orbital movement around the core and especially the spin movement).

\section{III.3. Fundamental Parameters in Electromagnetism}

These parameters can be seen in Fig. 6 .

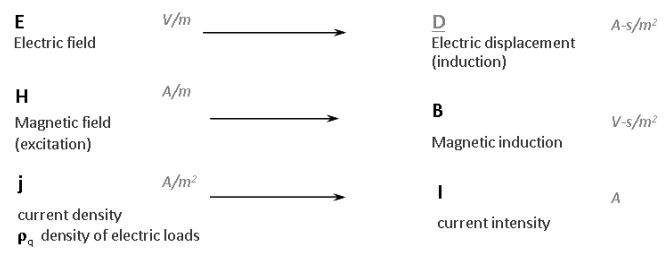

Fig. 6. Fundamental parameters in electromagnetism

\section{III.4. Maxwell Equations}

Basic relations between electric and magnetic parameters are described by Maxwell's equations, presented here under differential form:

- Coupling equations:

$$
\left\{\begin{array}{l}
\nabla \wedge \vec{E}=-\frac{\partial \breve{B}}{\partial t} \text { Induction law of Faraday } \\
\nabla \wedge \breve{H}=\vec{j}+\frac{\partial \vec{D}}{\partial t} \text { Ampere law corrected }
\end{array}\right.
$$

\section{- Conservation equations:}

$$
\left\{\begin{array}{l}
\nabla \cdot \breve{B}=0 \text { Gauss law for magnetism } \\
\nabla \cdot \vec{D}=\rho_{q} \text { Gauss law for electrostatics }
\end{array}\right.
$$

Field parameters $H$ and $B, E$ and $D$, as presented above, are linked by the materials coefficients $\mu$ and $\varepsilon$.

$$
\left\{\begin{array}{l}
\breve{B}=\mu \breve{H}\left(+\breve{B}_{r}\right) \text { magnetic properties } \\
\vec{D}=\varepsilon \vec{E}\left(+\vec{P}_{e}\right) \text { dielectric properties }
\end{array}\right.
$$

\section{III.5. Conduction and Propagation}

Maxwell equations make explicit that energy can be transmitted in space without the support of any medium.

Maxwell's coupling equations can be combined, under restrictive hypotheses in order to separate electric and magnetic parameters; it then leads to the following differential equation for a given field $C$ :

$$
\Delta C=\mu \sigma \frac{\partial C}{\partial t}+\mu \varepsilon \frac{\partial^{2} C}{\partial t^{2}}
$$

Depending on the values of the physical constants $\varepsilon, \mu, \sigma$, and especially of the ratio $\sigma / \varepsilon$, the dominating phenomenon can be:

- a diffusion (三 conduction), when the first degree term in the equation is predominant;

- a wave propagation, when the second degree term in the equation is predominant.

Furthermore, if electromagnetic phenomena are of sinusoidal nature $\left(C=C_{0} \sin \omega t\right)$, which is a quite frequent case in practical, the condition on the physical parameters can be linked to frequency $\mathrm{f}$ and to angular velocity $\omega=2 \pi f$ :

- diffusion $\equiv$ low frequencies

- propagation $\equiv$ high frequencies

- if $\omega<<\sigma / \varepsilon$, the main phenomenon is diffusion (conduction). This is applied to low frequencies and to very conductive media

- if $\omega>>/ \varepsilon$, the main phenomenon is propagation. This is applied to high frequencies and to insulating materials.

The transmission of electrical energy may be based 
either on conduction or on propagation. Today electrical energy networks are based on conduction. It was the belief of Tesla that energy transmission could also be based on propagation. However, important obstacles can be encountered trying to follow this way. One of them is the problem of source directivity, as will be examined later.

\section{III.6. How to Make WPT}

The possibilities are summarised by the diagram in Fig. 7.

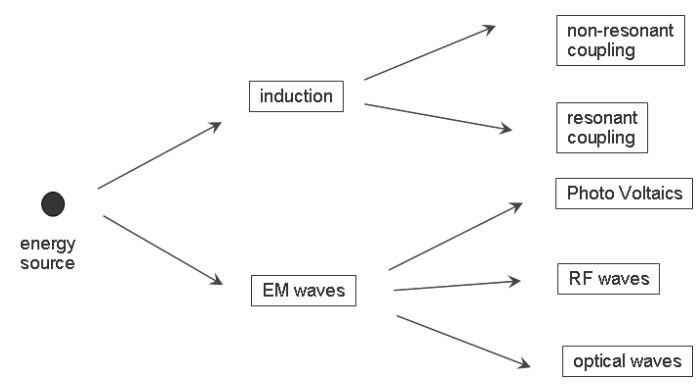

Fig. 7. The possible solutions for WPT

\section{III.6.1.Non Resonant Inductive Coupling}

The quite classical and well-known electrical transformer can be analysed as a wireless transmission device. In its most simple form, the transformer includes two windings with turn numbers $n_{1}$ (primary) and $n_{2}$ (secondary) respectively, strongly coupled by a magnetic circuit (Fig. 8). In conformity with Maxwell equations, the transformer first converts the electric energy of the primary winding into magnetic energy, circulating in the magnetic circuit as a magnetic induction flux $\Phi$. This magnetic flux is in its turn converted into electric energy in the secondary winding. The goal of this operation is to change the input voltage level $u_{1}$ into a different secondary voltage $u_{2}$ according to the relation:

$$
\frac{u_{2}}{u_{1}}=\frac{n_{2}}{n_{1}}
$$

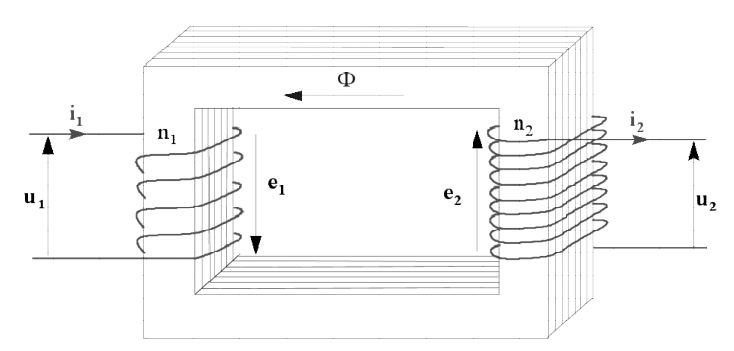

Fig. 8. Conventional electromagnetic transformer

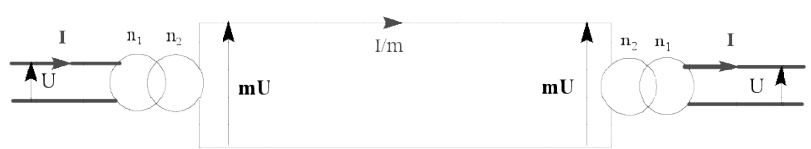

Fig. 9. Long distance transportation principle (high voltage $\mathrm{mU}$, low current $\mathrm{I} / \mathrm{m}$ )
This makes it possible to change low voltage into high voltage, in order to allow energy transmission at long distance ; reverse conversion of high voltage into low voltage is done at the receiving end. The transformer makes use of the magnetic induction principle (Faraday's induction law). It puts in evidence a fundamental property, which is the importance of frequency $f$ : simply, the higher the frequency is, the more induced voltage is collected :

$$
e=-\frac{d \phi}{d t} \Rightarrow E=\omega \Phi
$$

with $\omega=2 \pi f$ in harmonic regime. A part of the difficulties encountered by Tesla is his time was because he could hardly use high frequencies, such as the ones of micro-waves. Some of the actual approaches of WPT consist in an extrapolation of the transformer principle, simply increasing the distance between the two windings, and suppressing the magnetic core.

\section{III.6.2. Resonant Inductive Coupling: Basic Calculations and Experiments}

The transformer implements a very strong coupling, close to 1 , due to the presence of the magnetic core. The calculation of the coupling coefficient $k$ between two distant coils, in the air or in vacuum, leads on the contrary to weak values such as $10^{-2}$ or $10^{-3}$. In order to compensate this, the remedy is to use resonant coupling, i.e. coupling between tuned oscillating circuits. Among the studies devoted to the resonant coupling approach, [2] brings especially interesting results. One has compared theoretical calculations, numerical Spice simulations, and experimentation. Good agreement has been observed between all these. It has been confirmed that energy transmission has not been impeded by a nonconductive obstacle between the coils. In Fig. 10, a typical schematics for a resonant system is shown. Such a system includes four poles, in the complex plane, which correspond to a double frequency resonance.

Resistances in the system introduces damping, they reduce the resonances' quality factors, but also limit the overvoltage on the charge resistor. Experimentation has confirmed the practical difficulty to achieve an optimal tuning of L-C circuits for efficient energy transmission.

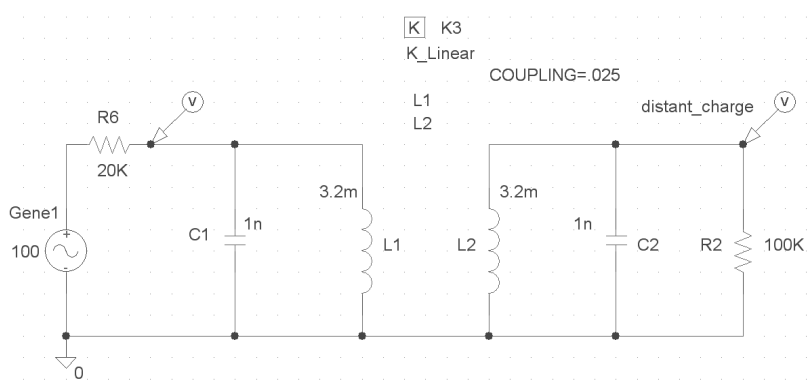

Fig. 10. Schematics for WPT through coupled circuits 


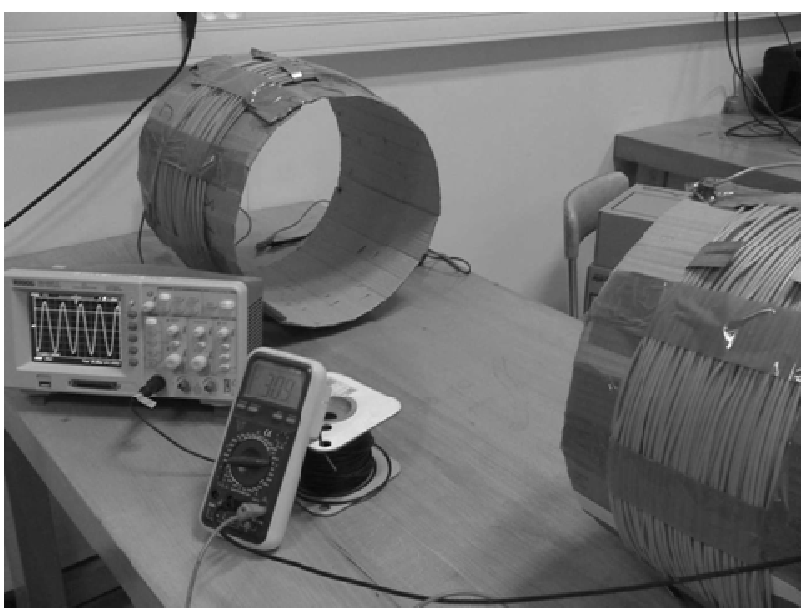

Fig. 11. Experimental setup used for study [2]

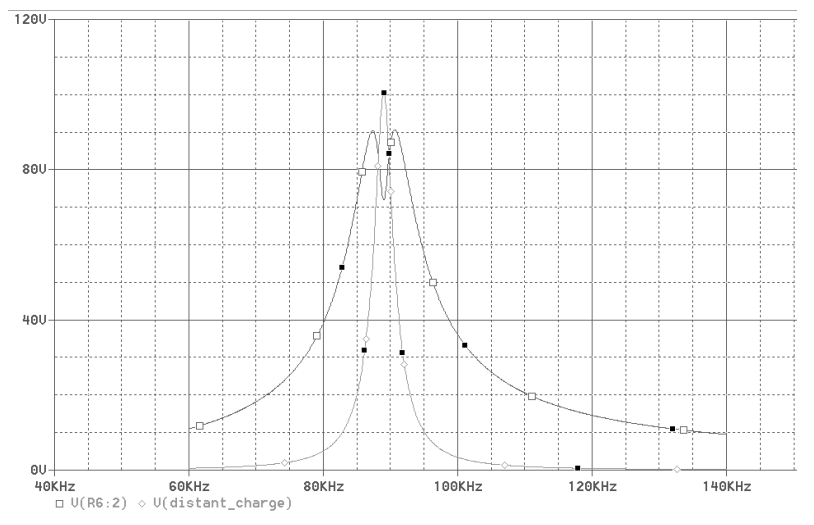

Fig. 12. Sample of simulation results for a given set of parameters, primary voltage in dark, and secondary voltage in clear

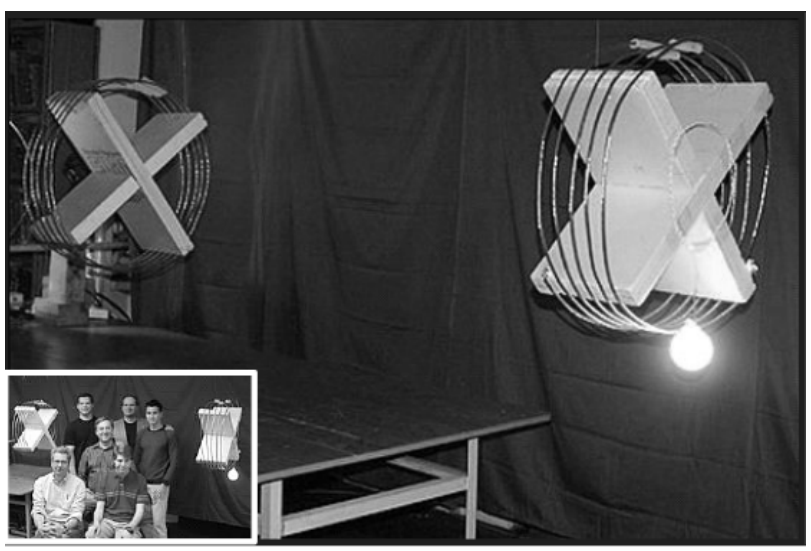

Fig. 13. Demonstration test bench and development team of Prof. M. Soljacic [4]

When the right adjustment can be found, then the efficiency can be very good. It should never be forgotten that as, the frequency increases, conventional schematics used for simulation have to be completed to integrate parasitic elements (inductances, capacitors, ...).

\section{III.6.3. The WiTricity Concept}

Resonant coupling between distant coils was successfully investigated at Massachusetts Institute of Technology (MIT) by the team of Professor M. SOLJACIC in 2006 [3].

Experimental conditions were the following:

- coils distance $=2 \mathrm{~m}$

- coils size : $0.6 \mathrm{~m}$ in diameter

- frequency $10 \mathrm{MHz}$

- power transmitted $60 \mathrm{~W}$

The efficiency obtained was $45 \%$ (90\% when distance is reduced to $1 \mathrm{~m}$ ).

Following this successful demonstration, company WiTricity Corp. was founded in 2007, in order to participate in the commercial development of applications of this technology.

\section{III.7. The Use of Propagation (High Frequencies)}

Propagation of electromagnetic waves may be envisaged as well for transmission of both energy and information. It is not necessary to recall the impressive development achieved today by communication systems: broadcasting, television, mobile phones, Wi-Fi computers networks, radar, and so on. Tesla was interested by the two application domains, but he was much more confident in conduction than in propagation.

A point source of electromagnetic energy propagates according to a spherical pattern (Fig. 14). Hence the energy density, measured by the Poynting vector $S_{p}$, decreases as $1 / r^{2}, r$ being the distance of a given point to the source, and $P$ the power of the source:

$$
S_{P}=\frac{P}{4 \pi r^{2}}
$$

At long distance, the ratio between the electric field $E$ and the magnetic field $H$ tends to a constant value, which is the wave impedance in the medium considered $Z=E / H$, with the value $Z=377 \Omega$ for vacuum or air. Waves can then be approximated as plane waves (Fig. 15). The wave dispersion is acceptable in the communications domain, because a wave receiver only needs a very small energy to catch the signal, and afterwards it amplifies this signal.

On the contrary, the spherical dispersion of the waves would lead to a non acceptable efficiency in the application to energy transmission. Hence the use of very directive antennas at emission and at reception to limit this effect (Fig. 16). A parabolic reflector in particular will be efficient to obtain a high directivity in the microwave band. Comparisons between transformer structures used in WPT are presented by [5]. A small power WPT system using a capacitive coupling is described by [6].

\section{III.7.1. The Frequency Bands}

To a wave frequency $v$ corresponds:

a photon energy $E=h v$, (with $h=6.626070 \times 10^{-34}$ J s, the Planck constant),

a wavelength $\lambda=\chi / v$ in a given medium with wave 
celerity $\chi$.

Celerity of electromagnetic waves in vacuum is $c=3 \times 10^{8} \mathrm{~m} / \mathrm{s}$ (celerity of light). Frequency bands of electromagnetic waves correspond to different physical phenomena, such radio communications, visible light, etc. (Fig. 17). Microwave frequency bands cover three decades. They correspond respectively to:

- Wavelengths from $1 \mathrm{~mm}$ to $1 \mathrm{~m}$,

- Frequencies from $300 \mathrm{GHz}$ to $300 \mathrm{MHz}$,

- Photon energies from $1.24 \mathrm{meV}$ to $1.24 \mu \mathrm{eV}$

Moreover, some subparts of these bands are allocated for given applications. For instance, in the microwave band, a specific band around frequency $2.45 \mathrm{GHz}$ is dedicated to Industrial, Scientific, and Medical applications (ISM band). This band may be used for energy transmission.

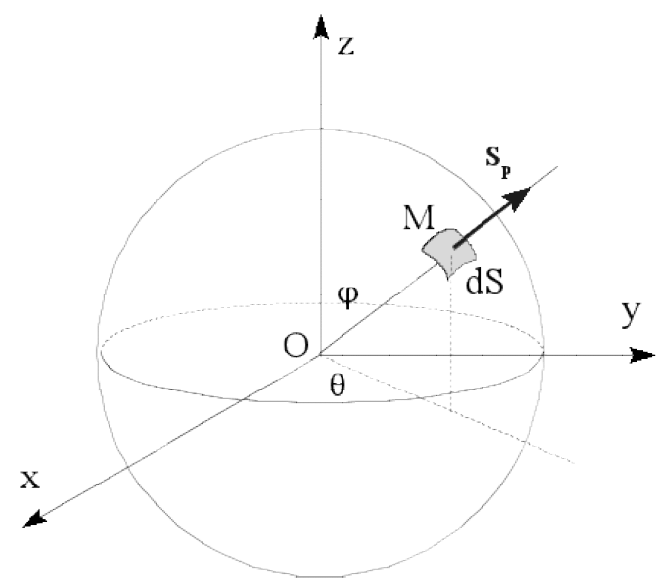

Fig. 14. Spherical radiation pattern of an isotropic source

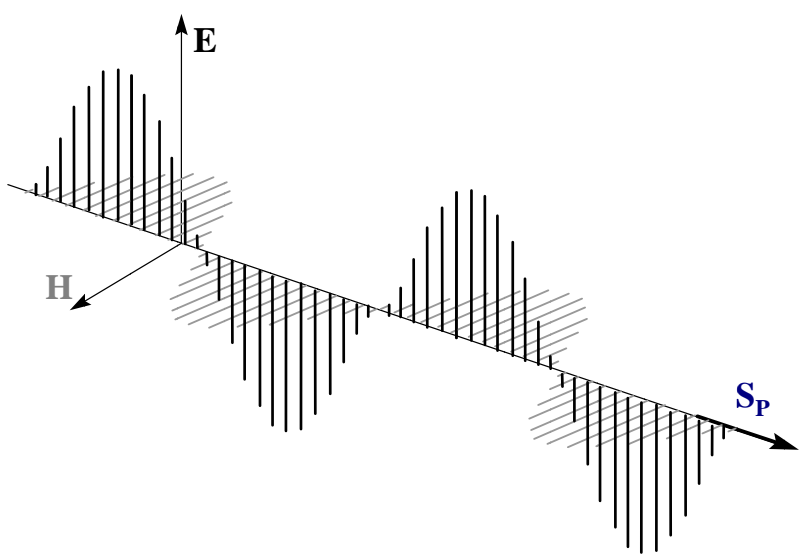

Fig. 15. Electromagnetic plane wave and associated Poynting vector $S_{p}$

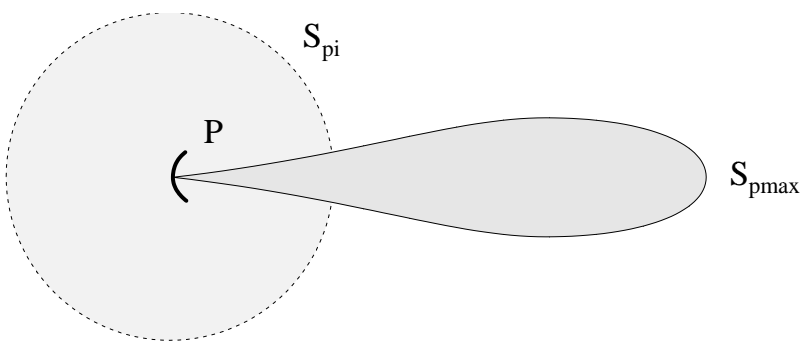

Fig. 16. Directive emission with use of parabolic reflector

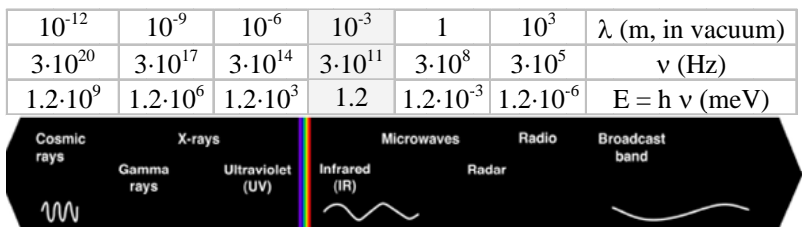

Fig. 17. The frequency bands

\section{III.8. Energy Transfer: Reception}

The reception of energy transmitted by WPT can use 3 techniques: Photo Voltaic, Radio-Frequency waves, or light waves. The photovoltaic effect, shown by some semiconductor materials, allows converting directly light into electric power. It may be explained by quantum mechanics. The energy of an electromagnetic radiation is quantized in photons, with individual energy hv (Fig. 18). The photovoltaic effect takes place at the junction of two semiconductors of respective dopings $\mathrm{P}$ and $\mathrm{N}$.

These semiconductors are present in a PV cell. When an individual photon interacts with an electron at the valence band of the semiconductor, the energy of the photon and the photon itself can be absorbed by the electron, then promoted to the conduction band, and leaving a hole in the valence band. This process generates an electron-hole pair. This is only possible when the photon energy is at least equal to the energy of the band-gap, i.e. the energy distance between the conduction and the valence bands. The population of so generated electrons and holes is then driven by the electrical field in the depletion zone of the PN junction, and can contribute to a voltage and to the corresponding current, when an electric load is connected to the PV cell.

Both the photo-voltage and the photo-current created here are DC magnitudes, and their product yields directly the electrical power converted by the PV cell.

- The theoretical limit for efficiency is around $41 \%$ for single junction cells, and $87 \%$ for multiple junction cells.

1) electrical antenna "rectenna"

The so-called rectenna includes an electrical rectifier associated to an electromagnetic antenna. The principle is the direct conversion of light into electricity using a rectifier working at radio or microwave frequencies. No quantum mechanics is involved here, since the electrons of the metallic antenna are already in the conduction band, and do not need to be promoted in energy by absorbing photons from the electro-magnetic radiation.

The phenomenon is a classical electromagnetic interaction between the electrons in the antenna, and the electric field of an incident wave.

In order to be efficient, the wavelength of the Electro Magnetic incident wave should match with the antenna length, in order to induce a resonant electrical current in the antenna.

At the difference of a PV cell, an antenna will generate at its output both an $\mathrm{AC}$ voltage and an AC current. A rectifier will thus be needed to transform the AC quantities into DC quantities. 


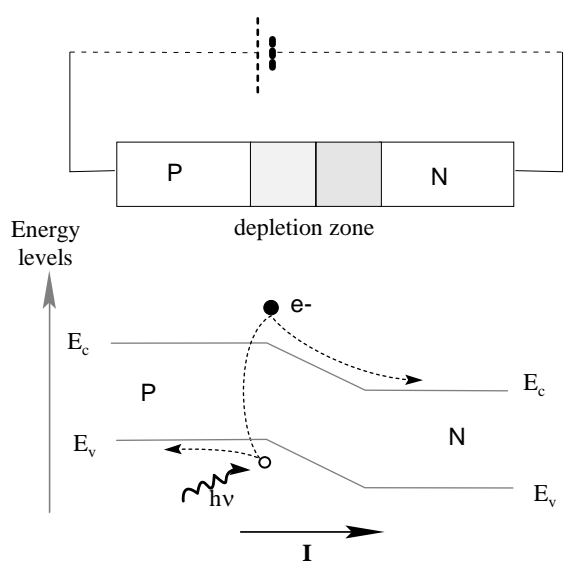

Fig. 18. Photovoltaic junction

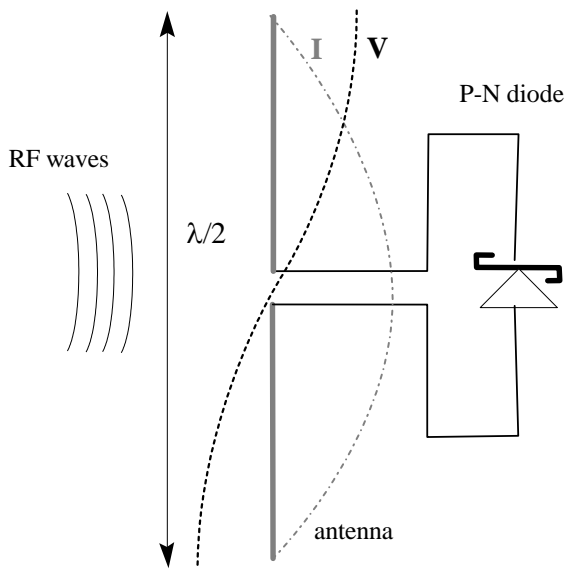

Fig. 19. Antenna in the plane of electric field E

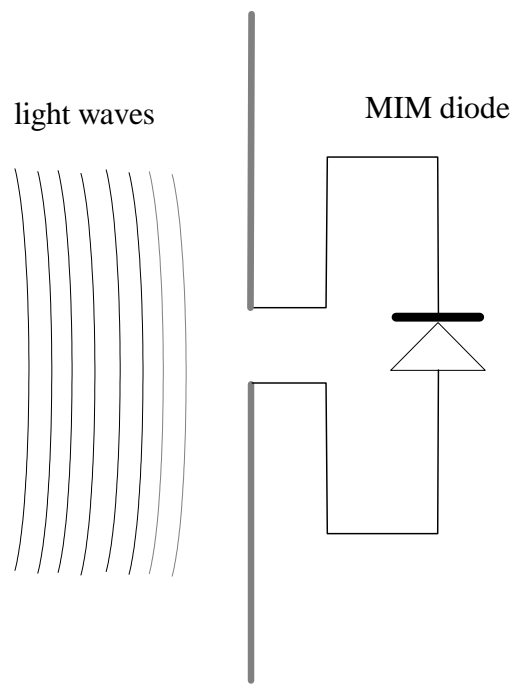

Fig. 20. Nanoantenna

A Schottky-type diode is especially adapted here, due to its short recovery time.

- The theoretical limit for efficiency is around $85 \%$, and a practical efficiency of $75 \%$ has been observed for some devices. An example of a rectenna structure with good performance is given by [7].

Electronic circuits to be associated with rectennas for low power applications have been studied especially by [8].

2) optical antenna

There is an apparent similarity between the optical antenna technology, and the rectenna technology.

However, profound differences exist.

The frequency of the electromagnetic radiation involved are here situated in the optical range, and the antenna has very small dimensions, corresponding to the wavelength domain ("nano-antenna") Rectifiers associated with optical rectennas should be operational at optical frequencies. Special types of diodes, typically Metal-Insulator-Metal (MIM) diodes are used. Moreover, specific phenomena such as plasmon resonances occur here.

- The theoretical limit for efficiency is around $85 \%$.

\section{Context Elements}

\section{IV.1. Technical Evolutions}

What are the technical evolutions, which permitted progress of WPT since Tesla's time? These evolutions are many, and mainly concern the electronic technologies. Especially the following should be retained:

- in the domain of signal electronics:

1) solid state semiconductors, initially under the form of discrete components (diodes, transistors) progressively replaced former vacuum tubes

2) integrated circuits gathered with increasing densities the elementary semiconductor elements

3) higher physical performances were attained concerning frequency domain, admissible voltage and current

4) very low supply power was made possible by semiconductor technologies such as Field Effect Transistor (FET) and Metal Oxide Semi Conductor (MOS and C-MOS)

- in the domain of power electronics:

It is showed in parallel equivalent progress; the first generation of power components (thyristors, transistors), have been progressively replaced by GTO (Gate Turn Off) and IGBT (Insulated Gate Bipolar Transistors). These have much higher specifications, and are simpler to use. These power components have facilitated the development of very high power rectifiers and inverters, today used for DC long distance energy transmission, up to several GW.

\section{IV.2. Development Milestones (Up To 2010)}

The stories of Wireless Power Transmission (WPT) and Space-based Solar Power (SSP) are closely associated. SSP took a concrete start in 1968. Main milestones are (list limited to most marking events):

- 1941: Science-fiction author Isaac Asimov describes, in a novel, a space-based power supply system;

- 1964: a microwave-powered helicopter model is 
made by W.C. Bron;

- 1968: presentation of the concept of Space-based Solar Power (SSP) by Dr. Peter Glaser (USA);

- 1973: first patent granted to Dr. Peter Glaser, foreseeing the use of microwaves, and introducing the concept of the rectenna;

- 1976: experimental transmission of $30 \mathrm{~kW} \mathrm{DC}$ at 1,6 $\mathrm{km}$ distance, by Jet Propulsion Lab - Goldstone, USA: in this experiment, a 26-m diameter parabolic antenna and a $450 \mathrm{~kW}$ klystron were used for emission at $2.388 \mathrm{GHz}$, while reception occurred through a $24 \mathrm{~m}^{2}$ antenna array [9];

- 1994: experiment by USAF using a low earth orbit satellite;

- 1995: start of NASA research studies;

- 1997: demonstration of WPT by microwaves at Grand-Bassin, La Réunion (France) by G. Pignolet: a demonstrator using a magnetron for microwave generation, a horn antenna for emission, and a parabolic receiver could light up a $200 \mathrm{~W}$ bulb at a distance of $700 \mathrm{~m}$; followed by further works [10];

- 1998: start of Japan Spatial Agency (NASDA) research studies, still under course today;

- 2001: NASDA announcement of plans for prototyping satellites of $10 \mathrm{~kW}$ and $1 \mathrm{MW}$ power levels;

- 2003: studies of the European Space Agency (ESA), presented to the French Senate assembly;

- 2008: demonstration of long distance WPT by microwaves $(20 \mathrm{~W}$ at $150 \mathrm{~km})$ in Hawaii by J.C. Mankins;

- 2009: creation of Japan consortium JAXA, including Mitsubishi and Space Agency;

- 2010: European space agency EADS Astrium plans to launch a demo satellite at level $10 \mathrm{~kW}$.

\section{Existing Applications}

\section{V.1. Microwave Oven}

Microwave oven is a kitchen appliance that heats food by exposing it to microwave radiation. This induces certain molecules in the food to rotate. Involved molecules have a significant dipolar moment (non isotropic electric charge diagram), especially water molecules, which orient themselves according to the direction of the electric field. Molecules movement produces thermal energy. This phenomenon is not a resonance. Microwave ovens heat foods quickly and efficiently because excitation is uniform in the outer part of a homogeneous, highly hydrous food item. Microwave ovens are today popular for reheating previously cooked foods, they are also useful for rapid heating of otherwise slowly prepared cooking items. The result obtained by such ovens differs from conventional cooking, due to the range of temperatures in play. The classical chemical Maillard reactions, necessary to obtain browning or baking, cannot take place. This application has been derived from radar technology developed during World War II. The generation of waves in the microwave range is obtained by a magnetron, which is a specific type of vacuum tube. The magnetron has a central cathode heated by a filament, no grid, and a mass and concentric anode in which several resonant cavities are hollowed out. An axial magnetic field is created by permanent magnets. The spiral path (due to the magnetic field) of the electrons is done at a frequency tuned to the resonant cavities. Because the magnetron is self-oscillating, it allows a very simple mounting in microwave ovens.

Available power is usually around $1 \mathrm{~kW}$, with an oscillation frequency of $2.45 \mathrm{GHz}$ thermal efficiency is about $65 \%$. In order to obtain these characteristics, an electrical voltage of about $2 \mathrm{kV}$ is necessary. The easiness of use of the magnetron has made microwave ovens popular for some experimentation facilities dedicated to WPT. However some precautions should be taken, not because of wave radiations, but because of the presence; first, of high values of voltage when $\mathrm{ON}$, and secondly, of large capacitors remaining charged with these voltages, even when OFF. Magnetron of higher power values are still used for radar applications.

\section{V.2. Passive RFID}

A RFID transponder (Radio Frequency IDentifier), also called tag, is placed in a given object and exchanges information with an external RFID reader. RFID systems can be either active or passive. Active RFID systems include a battery. Passive RFID systems collect from the external reader by means of an antenna a small energy quantity, sufficient for exchanging information. The signal sent by the reader is alternatively a modulated and non-modulated carrier. During non-modulated phases, the receiver stores the energy, in order to power up its internal circuitry. The small size of RFID chips makes is possible to place it almost everywhere. It has become mandatory for pet animals, and the debate is even under course about its possible application to human beings.
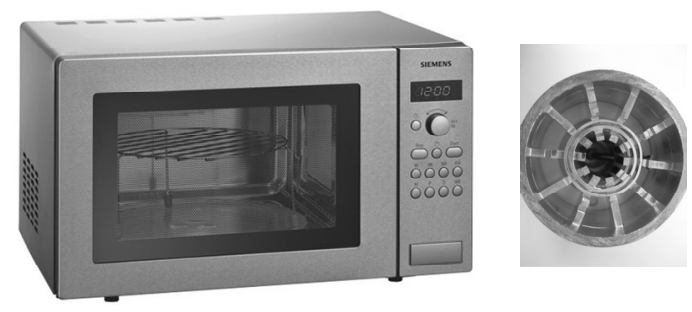

Fig. 21. microwave oven, and associated klystron for waves emission

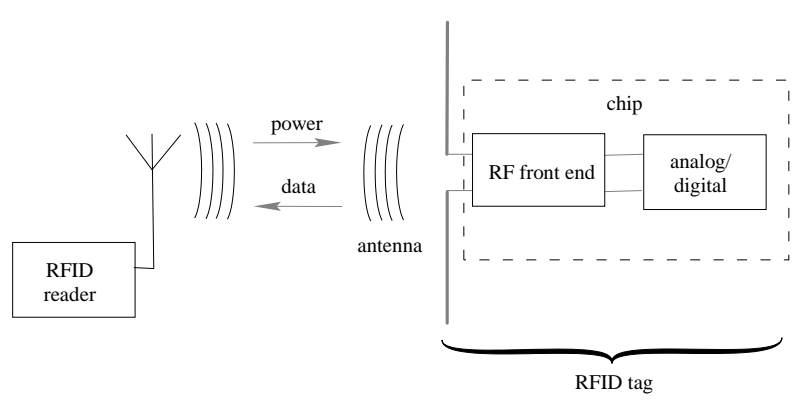

Fig. 22. RFID system 
The memory capacity of RFID chips is presently of 2 kilobytes. Several transmission frequencies are possible, from $125 \mathrm{kHz}$ to $5.8 \mathrm{GHz}$, different international standards such as ISO 14443 or ISO 15693 describe their utilisation. Lower frequencies allow a deeper penetration in the matter (skin effect), whereas higher frequencies allow faster information exchanges.

\section{V.3. Contactless Banking Card}

This is a current and popular application of RFID passive devices. This contactless device has exactly the size of a banking card. it includes integrated circuits for processing, and some non-volatile memory. It can communicate with a terminal via radio waves and also store and process data, depending on the quantity of memory embedded. It makes use of a transmission frequency of $13.56 \mathrm{MHz}$. In most countries, the use of this type of device is restricted to a small amount of money; however some countries set no limit. Economic studies state that the use of such cards result in a significant increase of the expenses realised. It may also be noted that miniature Lithium-Ion batteries are now available, which are small enough to be integrated in banking cards.
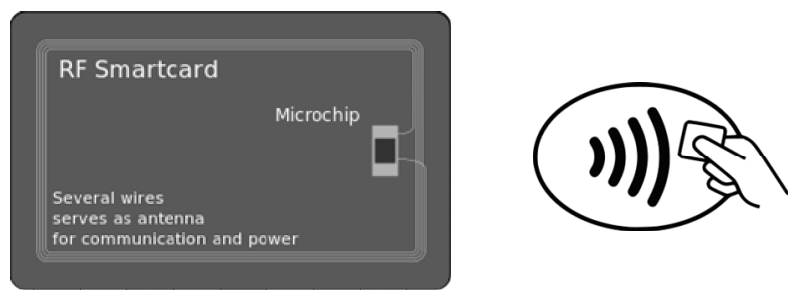

Fig. 23. Contactless banking card and associated symbol

\section{V.4. Induction Battery Chargers}

In the last decades, a quasi-infinite variety of applications and new devices based on new electronic components has appeared: computers, leisure electronics, mobile phones, etc. These potential applications have become effective ones, thus creating strong needs; some of us hardly imagine today life without all these modern tools. Unfortunately, and though the supply needs of electronics have strongly decreased; the problem of energy sources availability has often been faced, i.e. the problem of battery charging. Hence, some developers in the domain of WPT address the problem of battery charging, such as [11]. In some circumstances, the challenge of contactless battery loading may also be vital, such as in the biomedical field, the charge of human body internal prostheses. An Implantable Medical Device using automatic resonance tuning is described by [12]. Specific components for smartphone battery chargers are already available in the framework of the alliance between ST Microlectronics, main European semiconductor manufacturer, and Witricity Corp.

Although a known manufacturer of electronic devices recently renounced to his wireless charging device "Airpower" (2019), equivalent devices are today commercially available for smartphones and watches.

Similar devices exist for car battery charging, such as for Nissan Leaf and Toyota (up to $25 \mathrm{~kW}$ ). A three-phase vehicle charger solution has been studied by [14]. It can be observed that there is no decisive advantage for wireless charging compared to wired charging, because anyway a charging time is needed. On the contrary applications such as wireless banking cards and more generally, passive RFID applications, are more relevant because they enable faster transactions. Lastly, some other low-power applications of WPT have been studied, for instance the ones aiming at a better management of energy at home.

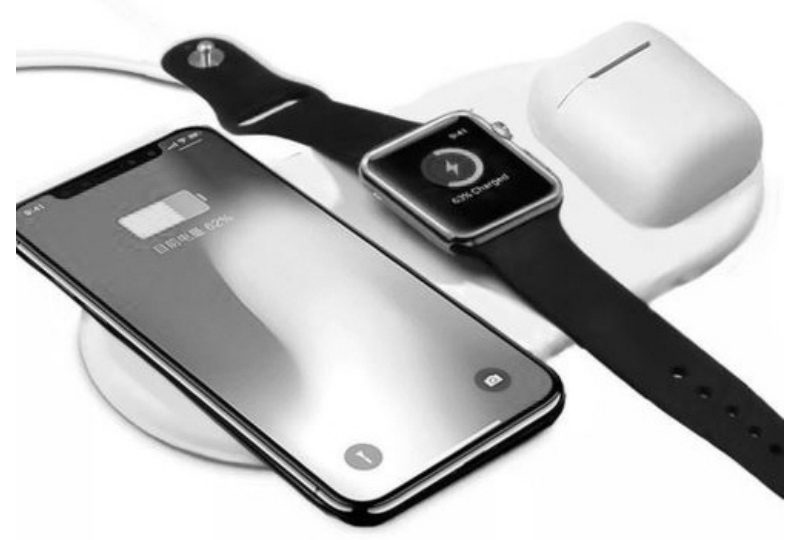

Fig. 24. Contactless smartphone and watch battery charging [13]

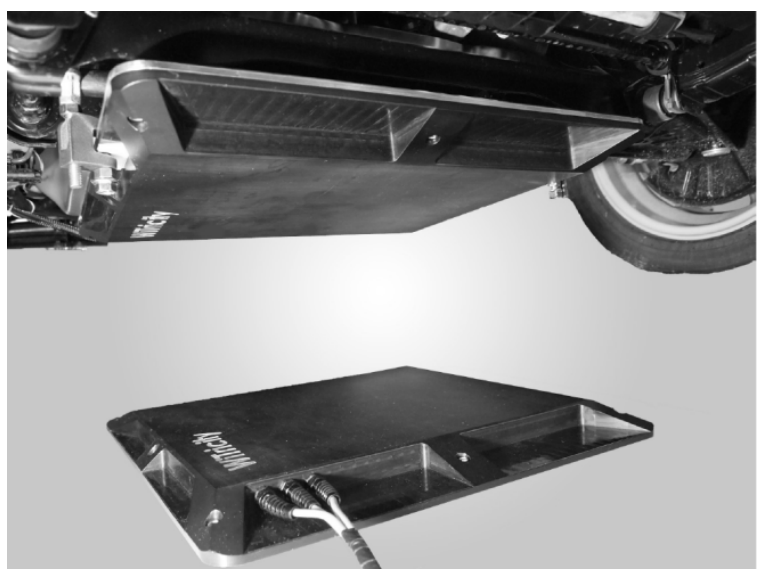

Fig. 25. Contactless car charger; source: WiTricity Corp

\section{Futuristic Applications}

\section{VI.1. What About Large Power at Long Distances?}

Today WPT has proved its feasibility under precise conditions, mainly short distance transmission and limited power ratings. However, WPT has been envisaged for another class of application, which is collecting solar power from space. Space-based Solar Power (SSP) would be a considerable challenge for mankind. It is a very abundant renewable energy; it 
would possibly solve a great part of environmental problems linked to the other forms of energy, even renewable ones. It is thus occasionally presented as attractive about anthropogenic climate change and fossil fuel depletion concerns. As implementing wires between earth and space would be unrealistic, one necessarily makes use of WPT. The application would implement an orbiting satellite, converting solar power into electricity by adapted photovoltaic panels.

The power would then be sent to the earth by an emitter/receiver set based either on a laser beam or through microwaves. The principle of collecting solar energy from space introduces a certain number of advantages compared to energy sources on the surface of the earth, but also creates huge drawbacks when compared with the same ones.

Advantages:

- radiation level received in space is about $50 \%$ higher than on earth surface (no atmospheric absorption and reflection, wider wavelength spectrum transmitted), and also more constant (no clouds, dust, weather events);

- collecting could be possible 24 hours a day (case of a sun-synchronous satellite);

The drawbacks are linked to:

- the considerable, and often unknown, costs of the equipments, mainly space equipments;

- their design, realisation, and implementation;

- the size of the receiving equipment needed on earth (such as $5 \mathrm{~km}^{2}$ of PV panels or mirror panels);

- lack of experience about large systems working in space: maintenance organisation, hazard linked to meteorites and "space debris", ...);

- possible safety issues due to human irradiation.

\section{VI.2. Technical Choices}

A set of technical choices is available at different steps of the implementation of SSP.

\section{VI.2.1. Energy Generation}

Solar energy, as well in space and on the earth, can be converted in electricity either by a Photo Voltaic process or by a thermal process (beam concentration by mirrors and condensable fluid thermo-dynamical cycle). While on the earth some power plants effectively already use the concentration process, the most studied process for implementation in space is Photo Voltaic.

\section{VI.2.2. Energy Transmission}

For transmission, microwave and laser may be envisaged:

\section{- Microwave transmission}

Microwave transmission in space would have the advantage of uninterrupted transmission through rain, snow, or any other atmospheric conditions and could provide gigawatts of power. Most small-scale experiments on earth made until now have made use of the microwaves.

\section{- Laser transmission}

Laser beam transmission in space would have the advantage of more simple reception devices, due to the high concentration of the beam. NASA has initially studied this process for space-to-space powering, and for powering a lunar basis (Selene project). The unit power per satellite would be in the range $1 \mathrm{MW}$ to $10 \mathrm{MW}$, a high number of satellites would hence be needed to transfer GW power levels. In addition, laser-transmitting satellites would have difficulty beaming power through clouds and rain. Despite recent progress of the lasers technique, especially pumped lasers, it seems that no concrete project to day is based on laser beams. [15]

\section{VI.2.3. Orbital Location of Satellites}

Different types of satellites can be envisaged:

\section{- Geostationary Earth Orbit (GEO) satellite.}

In this process, the geometry of the emitting antenna needs not to be adjusted; hence a simpler situation. GEO satellites may be replaced by Low Earth Orbit (LEO) or Medium Earth Orbit (MEO) satellites; in which case a whole network of satellites is required, exactly as for satellite communications, in order to keep in contact while they rotate around the earth.

- Sun-synchronous satellite.

Collecting would be possible 24 hours a day. Considering the huge difficulties anticipated, a wide number of variants has been imagined for implementation of SSP: construction in space, on the moon, or from an asteroid. Globally, cost predictions of all imagined solutions remain extremely difficult.

\section{VI.3. Energy Harvesting}

People are nowadays surrounded by many radiating electronic devices: radio, TV, mobile phone, wireless networks, detectors, remote controls of all kinds. In parallel, many new electronic devices require a very low level of supply. Energy harvesting, in the electrical domain, tries to exploit the conjunction of these two factors, collecting and recycling this energy.

\section{VI.3.1. What Can It Bring?}

Current levels of Electro Magnetic waves from existing applications can be seen in Table I. Collecting the ambient energy makes use of a specific rectenna. In fact, it is a rectenna array, designed to operate in a wide frequency band (2-18 GHz). Input power densities according to the table below range from $10 \mathrm{nW} / \mathrm{cm}^{2}$ to $0.1 \mathrm{~mW} / \mathrm{cm}^{2}$. The individual rectenna elements are spiral-shaped (see figure below) and distributed along as an array, with different orientations, in order to harvest energy from different polarized sources. Therefore, if an effective collecting area of $25 \mathrm{~cm}^{2}$ (on a panel of about $300 \mathrm{~cm}^{2}$ ) is considered, then associated input power will 
vary between $250 \mathrm{nW}$ and $2.5 \mathrm{~mW}$. If rectification efficiencies for such cases results to be $\eta_{250 \mathrm{nW}}=1 \%$ and $\eta_{2.5 \mathrm{~mW}}=20 \%$, then the output DC power harvested by this array will theoretically vary between $2.5 \mathrm{nW}$ and 500 $\mu \mathrm{W}$. Other examples of rectenna designs have achieved larger efficiencies, from $40-50 \%$ operating at $10^{-2}$ $\mathrm{mW} / \mathrm{cm}^{2}$ to $80 \%$ at $10 \mathrm{~mW} / \mathrm{cm}^{2}$ [16]. [17] describes another solution for harvesting based on a Frequency Selective Surface (FSS). [18] also presents a concrete realisation in order to improve harvesting systems.

Finally, Tesla was not wrong, thinking that there is energy available everywhere.

TABLE I

Current LeVEls OF ElECtro MaGNetic WAVES FROM EXISTING APPLICATIONS

\begin{tabular}{ccc}
\hline \hline Application Power density & $\mathrm{W} / \mathrm{m}^{2}$ & $\mathrm{~mW} / \mathrm{cm}^{2}$ \\
\hline FM radio at 50 km from a 100 kW base & $10^{-6}$ & $10^{-7}$ \\
station & $10^{-6}$ & $10^{-7}$ \\
ISM bands: Bluetooth & $10^{-5}$ & $10^{-6}$ \\
ISM bands: Wi-Fi & $10^{-5}$ to $10^{-4}$ & $10^{-6}$ to $10^{-5}$ \\
Current ambient level & $10^{-3}$ to $10^{-1}$ & $10^{-4}$ to $10^{-2}$ \\
Cellular phone at 50 m from base station & $10^{0}$ to $10^{+1}$ & $10^{-1}$ to $10^{0}$ \\
Solar Power Satellite Wireless Power & $10^{+3}$ & $10^{+2}$ \\
Transmission & & \\
Solar radiation in the visible range &
\end{tabular}

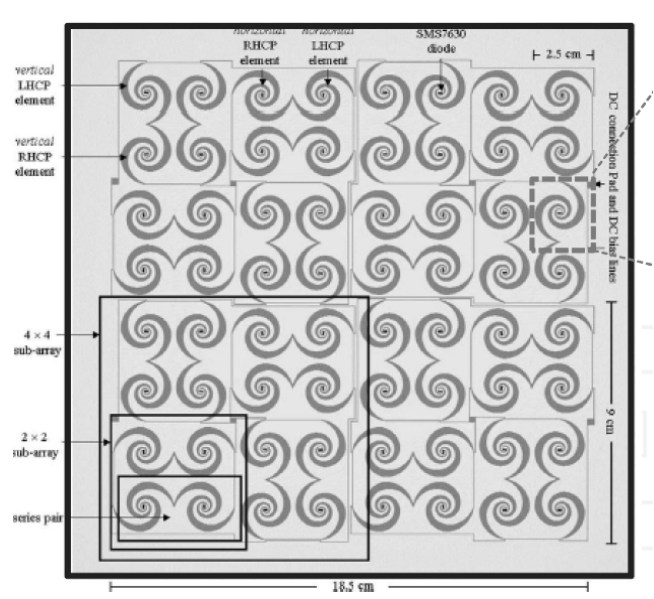

Fig. 26. Rectenna array for energy harvesting [16]

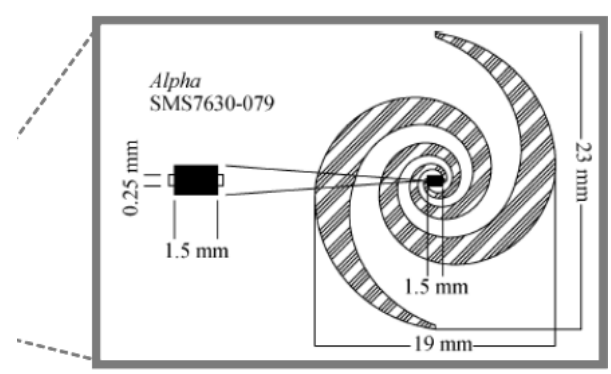

Fig. 27. Detail of a rectenna [16]

\section{VI.3.2. Exposure to Electromagnetic Waves}

The development of WPT could generate a fear that general exposure of humans to waves could increase. At the time being, most industrial countries have already implemented rules in order to define secure limits to exposure. These limits are detailed in technical standards such as EN 50360, EN 50361 in Europe, IEEE 1528 in the USA, and IEC 62209-1, IEC 62226-1, IEC 62233 international standards. [19]. Limits values depend on the circumstances, such as frequency bands, professional, or not exposure, part of the body exposed, etc. A thorough study of interferences has been published in [20]. [21] proposes a power control scheme to limit the exposition of humans to WPT. As an example, in Europe the permitted field level for exposure of the public are defined by European directive 199/519/CE (Table II). As it can be seen, for the microwave frequency band 2-300 $\mathrm{GHz}$ the value mentioned is $61 \mathrm{~V} / \mathrm{m}$, which is equivalent to a power density of $10 \mathrm{~W} / \mathrm{m}^{2}$. This level is currently attained by common electro-domestic appliances such as TV receivers or refrigerators. The power densities used for space transmission could attain of the order of magnitude of $1 \mathrm{~kW} / \mathrm{m}^{2}$.

TABLE II

EleCtric Field LeVEl (E), MAGNeTiC Field LeVEl (H) AND MAGNETIC INDUCTION (B) VALUES For DIFFERENT FREQUENCY BANDS

\begin{tabular}{ccccc}
\hline \hline & & & & Power \\
Frequency band & $\begin{array}{c}\text { Electric field } \\
\text { level E (V/m) }\end{array}$ & $\begin{array}{c}\text { Magnetic } \\
\text { ield level H } \\
(\mathrm{A} / \mathrm{m})\end{array}$ & $\begin{array}{c}\text { Magnetic } \\
\text { induction B B } \\
(\mu \mathrm{T})\end{array}$ & $\begin{array}{c}\text { equivalent } \\
\text { plane } \\
\text { wave } \mathrm{S}_{\mathrm{eq}} \\
\left(\mathrm{W} / \mathrm{m}^{2}\right)\end{array}$ \\
\hline $0-1 \mathrm{~Hz}$ & - & 32000 & 40000 & - \\
$1-8 \mathrm{~Hz}$ & 10000 & $32000 / f^{2}$ & $40000 / f^{2}$ & - \\
$8-25 \mathrm{~Hz}$ & 10000 & $4000 / f$ & $5000 / f$ & - \\
$0.025-0.8 \mathrm{kHz}$ & $250 / f$ & $4 / f$ & $5 / f$ & - \\
$0.8-3 \mathrm{kHz}$ & $250 / f$ & 5 & 6.25 & - \\
$3-150 \mathrm{kHz}$ & 87 & 5 & 6.25 & - \\
$0.15-1 \mathrm{MHz}$ & 87 & $0.73 / \mathrm{f}$ & $0.92 / f$ & - \\
$1-10 \mathrm{MHz}$ & $87 / \sqrt{ } f$ & $0.73 / f$ & $0.92 f$ & \\
$10-400 \mathrm{MHz}$ & 28 & 0.073 & 0.092 & 2 \\
$400-2000 \mathrm{MHz}$ & $1.375 \sqrt{f}$ & $0.0037 \sqrt{f}$ & $0.0046 \sqrt{f}$ & $f / 200$ \\
$2-300 \mathrm{GHz}$ & 61 & 0.16 & 0.20 & 10 \\
\hline \hline
\end{tabular}

\section{Research under Course}

From 2015 to now, although the great difficulties anticipated for the application of WPT to high power and long distance, most major industrial countries have not renounced to investigate the research field. The most active countries seem to be China and Japan.

- China

Official agencies, such as Xinhuan News, briefed a roadmap for a $1 \mathrm{GW}$ commercial system in 2050.

Chinese researchers consider they would need a 5 ton weight satellite, which does not exist today yet. However they also consider as realistic to have a first experimental spatial plant around 2030. The expected size of the receiver $\left(5 \mathrm{~km}^{2}\right)$ will be one of the major problems to handle.

- Japan

SSP has been established by law (2008) as a national goal for Japan. The JAXA consortium project (see above) is still under course. A prototype satellite orbiting is foreseen by 2030 . Two recent significant results have been announced (2015) :

a JAXA system could transfer $1.8 \mathrm{~kW}$ to a target 
receiver located at $55 \mathrm{~m}$.

- a Mitsubishi system could transfer $10 \mathrm{~kW}$ at $500 \mathrm{~m}$.

- United States

In a first period (1978), public offices DoE and NASA invested in a strong research effort, which was discontinued around 1980. At that time risks associated to SSP were assessed as much too high. In 1997, NASA undertook a new study, said "Fresh Look", to take in account technological progress realised in between. A specialised institution was then created (1999), the Space Solar Power Exploratory Research and Technology (SERT). This institution is still active today. Projects federating Defence, Diplomacy, and Development administrations (2015) currently receive great attention. Public actions are being launched on the Internet petitions site change.org so as to boost corresponding developments.

- Europe

No marking project is presently to be highlighted.

Many works and publications can regularly be found in literature.

Developments linked to SSP could also find application in other domains, such as space exploration (powering space vehicles).

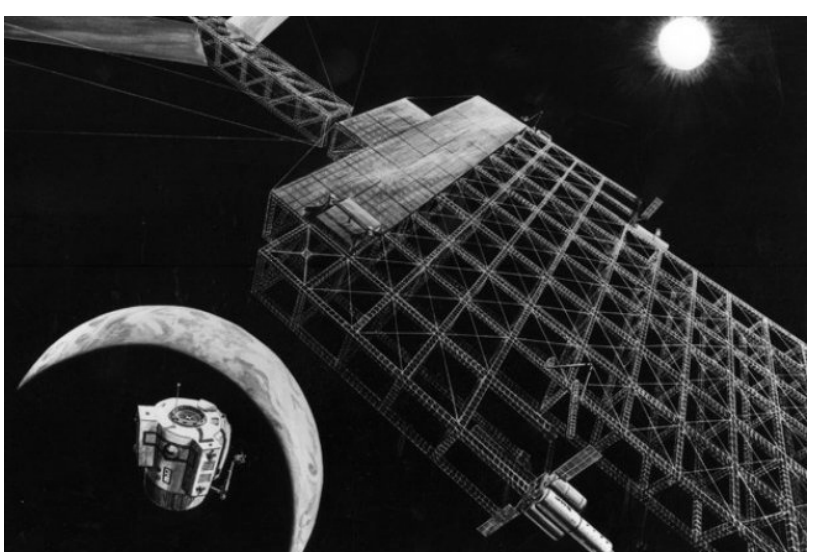

Fig. 28. A NASA overview for SSP

\section{Conclusion}

The concept of WPT (Wireless Power Transmission) is more than one century old. The concept of SSP (Space-based Solar Power) is almost half a century old.

Do we still have a long time to wait to see the emergence of corresponding achievements? The progress of technology and especially the one of electronic components has made many applications possible, which were nevertheless in the past considered as definitely unrealistic. WPT henceforth belongs to the domain of technology development. SSP remains a topic for longterm research. In the field of high power transmission, if still no effective implementation exists, no definitive obstacle on the road has been found out. Given the amount of energy involved, non expected uses of WPT and SSP systems are to be anticipated. Such are also their possible unexpected technical failures.

\section{Appendix I}

The case of a linear isotropic homogeneous medium, such as vacuum or air $(\varepsilon, \mu$, and $\sigma$ are constant in time, and scalar) is considered; if it is also supposed that no electric charges is present: $\rho_{q}=0$, then $\breve{B}=\mu \breve{H}$ and $\vec{D}=\varepsilon \vec{E}$, Maxwell coupling equations become quite symmetrical:

$$
\begin{aligned}
& \vec{\nabla} \wedge \vec{E}=-\mu \frac{\partial \vec{H}}{\partial t} \\
& \vec{\nabla} \wedge \vec{H}=\varepsilon \frac{\partial \vec{E}}{\partial t}+\sigma \vec{E}
\end{aligned}
$$

The general vector relationship written is used here for any given field $C$ :

$$
\vec{\nabla} \wedge(\vec{\nabla} \wedge \vec{C})=\vec{\nabla} \cdot(\nabla \vec{C})-\nabla^{2} \vec{C}
$$

that is applied for instance to the electrical field $\vec{E}$ :

$$
\begin{gathered}
\vec{\nabla} \wedge(\vec{\nabla} \wedge \vec{E})=-\mu \frac{\partial}{\partial t}(\vec{\nabla} \wedge \vec{H}) \\
\vec{\nabla} \wedge(\vec{\nabla} \wedge \vec{E})=-\mu \sigma \frac{\partial \vec{E}}{\partial t}-\mu \varepsilon \frac{\partial^{2} \vec{E}}{\partial t^{2}} \\
\vec{\nabla} \cdot \vec{E}=\frac{\rho_{q}}{\varepsilon_{0}} \\
\text { if } \rho_{q}=0 \quad \vec{\nabla} \cdot(\nabla \vec{E})=0
\end{gathered}
$$

It remains:

$$
\Delta \vec{E}=\mu \sigma \frac{\partial \vec{E}}{\partial t}+\mu \varepsilon \frac{\partial^{2} \vec{E}}{\partial t^{2}}
$$

Assuming wave celerity $\chi=\frac{1}{\sqrt{\varepsilon \mu}}$, in all cases it will be:

$$
\Delta C=\mu \sigma \frac{\partial C}{\partial t}+\frac{1}{\chi^{2}} \frac{\partial^{2} C}{\partial t^{2}} \text { with } C=E, B
$$

According to values of physical constants $\varepsilon, \mu, \sigma$, the predominant phenomena can be:

- a diffusion (conduction):

$$
\Delta C=\mu \sigma \frac{\partial C}{\partial t}
$$

- a wave propagation:

$$
\Delta C=\frac{1}{\chi^{2}} \frac{\partial^{2} C}{\partial t^{2}}
$$


If the electromagnetic phenomena are of sinusoidal nature $\left(C=C_{0} \sin \omega t\right)$, the condition on physical parameters becomes:

- diffusion (low frequencies):

$$
\omega<<\frac{\sigma}{\varepsilon}
$$

- propagation (high frequencies):

$$
\omega>>\frac{\sigma}{\varepsilon}
$$

In the last case, the equation becomes a Helmholtz vector equation: $\nabla^{2} C+\omega^{2} C=0$, with $C=E, B$.

Concerning WPT, it will be retained that the use of high frequencies will be adapted to long distance transmission.

\section{Appendix II}

Non-resonant coupling between two distant coils

The electromagnetic coupling between two identical circular windings, with radius $R$ is considered; they are assimilated to flat coils. These coils have the same axis, and they are placed at distance $D$ from each other. The first one is considered as a primary (excitation coil), the other one is considered as a secondary (receiver coil).

It is supposed that $D>R: D \cong D^{\prime}$.

The field in the coils axis is calculated to determine self inductances $L_{1}, L_{2}$ and mutual inductance $M$, and the coupling coefficient is derived from them; the two coils are identical:

$$
k=\frac{M}{\sqrt{L_{1} L_{2}}}=\frac{M}{L}
$$

Coupling is strong if $k>0.5$,coupling is weak if $k<0.5$. Biot and Savart elementary induction law is applied:

$$
d \breve{B}=\frac{\mu_{0}}{4 \pi} I d \vec{l} \wedge \frac{\vec{u}}{r^{2}}
$$

1) case of the emitter coil:

$$
\begin{gathered}
d B=\frac{\mu_{0}}{4 \pi} \cdot \frac{I d l}{R^{2}} \\
B=\frac{\mu_{0}}{4 \pi} \cdot \frac{1}{R^{2}} \int d l=\frac{\mu_{0} I}{2} \cdot \frac{1}{R}, \\
H=\frac{I}{2 R}
\end{gathered}
$$

This defines the field at centre of coil 1 along its axis. 2) case of the receiver coil:

$$
d B \approx \frac{\mu_{0}}{4 \pi} \cdot \frac{I d l}{D^{2}}
$$

The magnetic induction along the axis is obtained by projection:

$$
\begin{gathered}
d B_{x} \approx \frac{\mu_{0}}{4 \pi} \cdot \frac{I d l}{D^{2}} \cdot \frac{R}{D} \\
B_{x} \approx \frac{\mu_{0}}{4 \pi} \cdot \frac{I}{D^{2}} \cdot \frac{R}{D} \int d l=\frac{\mu_{0} I}{2} \cdot \frac{R^{2}}{D^{3}} \\
H=\frac{I R^{2}}{2 D^{3}}
\end{gathered}
$$

It can be observed that the axial field strongly decreases as a function of distance D.

- numerical application.

$R=0.30 \mathrm{~m}, f=11 \mathrm{MHz}, D=1$ to $10 \mathrm{~m}, L_{1}=1.48 \mathrm{mH}$, $Z_{1}=L_{1} \omega=102.3 \mathrm{k} \Omega$ (primary)

Values obtained for coupling coefficient $k$ are weak on the whole range of distances. Even with a high voltage at primary coil, transmitted energy will be small.

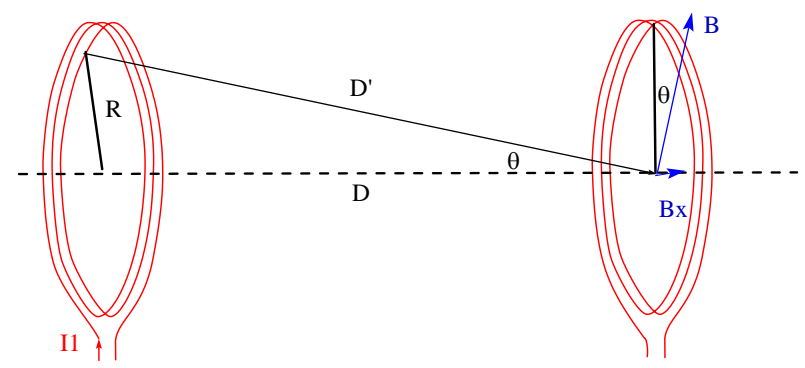

Fig. 1A. System of two coupled coils

\section{Acknowledgements}

An attentive reading of this text was made by $\mathrm{Mr}$. Samuel Nguefeu, RTE-France, Paris (France).

\section{References}

[1] Martin T. C., Tesla N. The Tesla Rotating Magnetic Field, Newage Science ed., ISBN Code 978-1425318734, (2005).

[2] Cabral M., Boriello-Goulart L., Marques T., Pontes-Gondim F., Wireless Power Transmission, École Centrale Paris, Project Report, (2009).

[3] Starck J. C. Wireless Power Transmission utilizing a phased array of Tesla coils, Dept of Electrical Engineering and Computer Sciences of Massachusetts Institute of Technology, Thesis, (2004).

[4] http://news.mit.edu/2007/wireless-0607

[5] Savu, A., Radoi, A., Florescu, A., Transformer Analysis for Wireless Power DC-DC Converters, (2014) International Review of Electrical Engineering (IREE), 9 (1), pp. 26-35.

[6] Yi, K., Capacitive Coupling Wireless Power Transfer Circuit with a Compensated L for High Voltage Gain and Soft Switching, (2018) International Review of Electrical Engineering (IREE), 13 (5), pp. 352-356. doi: https://doi.org/10.15866/iree.v13i5.15343

[7] Latrach $\mathrm{M}$. et al., A new design of a microstrip rectenna at 5.8 $\mathrm{GHz}$ for wireless power transmission applications, International Journal of Electrical and Computer Engineering, vol. $9 \mathrm{n}^{\circ} 2$, pp. 1258-1266, (2019). 
doi: 10.11591/ijece.v9i2

[8] Merabet B., Contribution to the study of power transmission at distance by microwaves, École Nationale Supérieure de Cachan, Thesis (2012), NNT : 2010DENS0048, pp. 21-30.

[9] Dickinson R.M. Performance of a high-power, $2.388 \mathrm{Ghz}$ receiving array in wireless power transmission over $1.54 \mathrm{~km}$, IEEE MTT-S International Microwave Symposium, pp. 139-141, (1976).

[10] Pignolet G. et al. Grand Bassin Case Study: an Original Proof-OfConcept Prototype for Wireless Power Transportation, WPTC Conference at Montreal, (2018). doi: 10.1109/WPT.2018.8639227

[11] Wang W., Study of the wireless power transmission based on magnetic coupled resonance, Montréal University thesis, (2014).

[12] Lee B., Kiani M., Ghovanloo M., A Triple-Loop Inductive Power Transmission System for Biomedical Applications, IEEE Transactions on biomedical circuits and systems, 2015. doi: 10.1109/TBCAS.2014.2376965

[13] https://fr.shopping.rakuten.com/offer/buy/3844114950

[14] Romba, L., Valtchev, S., Melicio, R., Three-Phase Magnetic Field Tested in Wireless Power Transfer System, (2016) International Review of Electrical Engineering (IREE), 11 (6), pp. 586-597. doi: https://doi.org/10.15866/iree.v11i6.9889

[15] Summerer L., Purcell O., Concepts for wireless energy transmission via laser, International Conference on Space Optical Systems and Applications ICSOS, (2009).

[16] Abadal G., Alda J., Agusti J., Electromagnetic Radiation Energy Harvesting - The Rectenna Based Approach, pp. 79-106, (2014). doi: http://dx.doi.org/10.5772/57118

[17] Nosrati M. et al. Parallel-series connected rectenna array using frequency selective surface (FSS) for power harvesting applications at $5.8 \mathrm{GHz}$, International Journal of $R F$ and Microwave Computer-Aided Engineering, (2019). doi: https://doi.org/10.1002/mmce.21819

[18] Aloulou, R., Lucas de Peslouan, P., Armand, J., Mnif, H., Alicalapa, F., Loulou, M., Lan Sun Luk, J., Micropower Clock Generator Circuit Using an Optimized Band-Gap Reference for Energy Harvesting Charge Pumps, (2015) International Review of Electrical Engineering (IREE), 10 (2), pp. 257-265. doi: https://doi.org/10.15866/iree.v10i2.5132

[19] Fanton J. P. Electro Magnetic Compatibility, Course textbook at École Centrale Pékin, China, vol. 10, pp. 10-11, (2011)

[20] Baikova, E., Valtchev, S., Melicio, R., Krusteva, A., Pires, V., Study of the Electromagnetic Interference Generated by Wireless Power Transfer Systems, (2016) International Review of Electrical Engineering (IREE), 11 (5), pp. 526-534. doi: https://doi.org/10.15866/iree.v11i5.9773

[21] Seong-Min Kim et al. Advanced Power Control Scheme in Wireless Power Transmission for Human Protection From EM Field, IEEE Transactions on Microwave Theory and Techniques, vol. 63, n 3, pp. 847-856, (2015).

doi: 10.1109/TMTT.2015.2398444

\section{Authors' information}

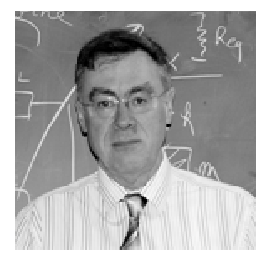

J. P. Fanton received the Dipl. Ing. degree from Ecole Supérieure d'Electricité, today Centrale Supelec (France). Since 2005 he is a lecturer at ECAM-EPMI, Cergy-Pontoise (France). He is a member of the Scientific Committee for Electricity and Magnetism at Laboratoire National d'Essais (LNE), Paris, France. 